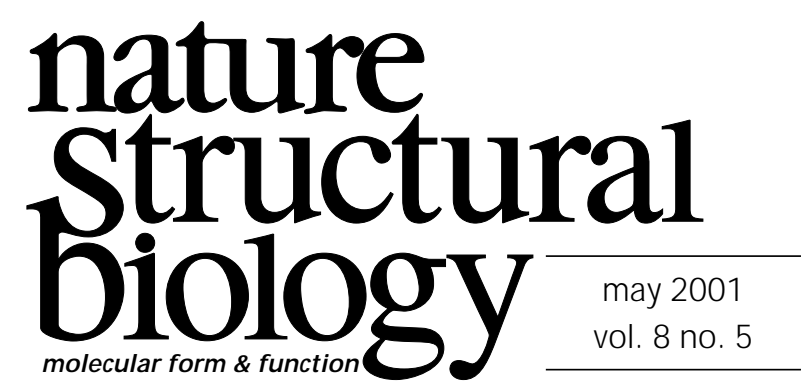

\section{A collection of History}

The History section of Nature Structural Biology was launched in January, 1998. Since then, we have presented a short History report every month, written by either one of the editors or a scientist in the field. Over the past few years, historical reports of this kind have become more frequent in other scientific journals and on websites around the world. We hope the History pieces in our journal have served as useful brief introductions to the early literature in a field or - at the very least - that they have been enjoyable diversions in a publication dedicated primarily to the presentation and analysis of detailed research reports. As a service to our readers, we have decided to compile what we think are some of the most interesting History pieces into an online archive that is accessible from the Nature Structural Biology home page.

History pieces written by the editors have ranged broadly for example, they have included a short biography of Anders Jonas Angstrom, a meeting report about a symposium to celebrate the $50^{\text {th }}$ anniversary of the Biomolecular Structure Laboratory at Birkbeck College, a description of the discovery of restriction enzymes, and a report on some of the first cryo-electron microscopy experiments. However, undoubtedly the most valuable reports in the History section are those that have been written by scientists who were present at the time of an interesting discovery, or who have specific and/or first-hand knowledge about the events described. These pieces represent a special kind of 'received wisdom' that cannot be gleaned from reading the papers that present the research itself, and they are a valuable resource for those interested in the oral history of structural and molecular biology. Anyone who may have missed these reports or anyone who would like to enjoy them again may find them in the History collection on our web site.

The reports in the collection are an eclectic bunch. Gary Yellen describes some of the experiments on ion channel gating that foreshadowed the first structure of an ion channel. Tom Blundell speaks of his time in Dorothy Hodgkin's laboratory in the 1960s. Alex Rich talks about the discovery of the collagen structure. Louise Johnson recalls a special lecture by David Phillips on the structure of lysozyme. Robert (Buzz) Baldwin summarizes the protein folding field before the days of 'molten globules'. Art Horwich remembers working with Paul Sigler on the structure of GroEL. Jane Richardson describes how the common 'ribbon diagrams' to depict molecular structures evolved. Sidney Altman explains how he began work that led to the discovery of RNase P. Arthur Edison summarizes Linus Pauling's insight into the planarity of the peptide bond. Nina Federoff describes how transposons were first discovered. And, finally, Michael Levitt (also on page 392 of this issue) gives insight into the early days of computer simulations of protein and nucleic acid structures.

If you haven't read these History reports - you should. They are entertaining and informative, and in many cases provide rare glimpses of the scientists behind the science. We hope to add equally engaging accounts to our History collection in the future. 